\title{
Biomarcadores clínicos de sintomas não motores na doença de Parkinson
}

\author{
Clinical biomarchers of non-motor symptoms \\ in Parkinson's disease
}

\section{Biomarcadores clínicos de síntomas no motores en la enfermedad de Parkinson}

\author{
Robson Arruda Souzaㄹ, Ivan Victor Torres Vieira², \\ Jairo Pereira da Silva²
}

\begin{abstract}
1.Fisioterapeuta, Mestre, Especialista em Fisioterapia em Geriatria e Gerontologia, Departamento de Medicina - Centro Universitário UNIFACISA, Campina Grande-PB, Brasil. 2.Graduando em Medicina, Departamento de Medicina - Centro Universitário UNIFACISA, Campina Grande-PB, Brasil.
\end{abstract}

\begin{abstract}
Resumo
Introdução. A doença de Parkinson (DP) é o segundo distúrbio neurodegenerativo que mais afeta a população idosa acima de 60 anos. Os sintomas não motores desempenham um papel fundamental na trajetória da doença, assim a identificação de biomarcadores periféricos e/ou centrais que sejam sensíveis, específicos e econômicos para o diagnóstico diferencial precoce, prognóstico e tratamento da DP são fundamentais para o manejo desses pacientes. Objetivo. Identificar e discutir as características dos principais de biomarcadores clínicos de sintomas não motores na DP. Método. Trata-se de uma revisão bibliográfica através das bases dados PubMed/MEDLINE, SciELO, Science Direct. Os artigos foram selecionados segundo os critérios de inclusão: ensaios clínicos controlados randomizados, estudos quasi-experimentais e revisões sistemáticas que registraram a utilização de biomarcadores de sintomas não motores na DP. Resultados. A estratégia de busca utilizada resultou em 705 títulos, onde um total de 21 estudos foram incluídos para análise. Mais de $90 \%$ dos pacientes experimentam sintomas não motores durante o curso da DP. Os principais sintomas não motores relatados envolvem alterações do sistema sensorial, cognitivo e mudanças de personalidade. Conclusão. Os biomarcadores identificados neste estudo destacam o papel da identificação dos sintomas clínicos não motores no diagnóstico precoce da doença de Parkinson e o entendimento da etiologia para direcionar um manejo eficaz.
\end{abstract}

Unitermos. Biomarcador; Doença de Parkinson; Avaliação de Sintomas

\begin{abstract}
Introduction. Parkinson's disease (PD) is the second neurodegenerative disorder that most affects the elderly population over 60 years. Non-motor symptoms play a fundamental role in the disease trajectory, so the identification of peripheral and / or central biomarkers that are sensitive, specific, and economical for the early differential diagnosis, prognosis and treatment of PD are essential for the management of these patients. Objective. Identify and discuss the characteristics of the main clinical biomarkers of non-motor symptoms in PD. Method. This is a bibliographic review using the databases PubMed / MEDLINE, SciELO, Science Direct. The articles were selected according to the inclusion criteria: randomized controlled clinical trials, quasi-experimental studies and systematic reviews that recorded the use of biomarkers of non-motor symptoms in PD. Results. The search strategy used resulted in 705 titles, where a total of 21 studies were included for analysis. More than $90 \%$ of patients experience nonmotor symptoms during PD. The main non-motor symptoms reported involve changes in the sensory, cognitive system and personality changes. Conclusion. The biomarkers identified in
\end{abstract}


this study highlight the role of identifying non-motor clinical symptoms in the early diagnosis of Parkinson's disease and understanding the etiology to guide effective management.

Keywords. Biomarker; Parkinson's Disease; Symptom Assessment

\begin{abstract}
Resumen
Introducción. La enfermedad de Parkinson (EP) es el segundo trastorno neurodegenerativo que más afecta a la población anciana mayor de 60 años. Los síntomas no motores juegan un papel fundamental en la trayectoria de la enfermedad, por lo que la identificación de biomarcadores periféricos y / o centrales sensibles, específicos y económicos para el diagnóstico diferencial precoz, pronóstico y tratamiento de la EP son fundamentales para el manejo de estas. pacientes. Objetivo. Identificar y discutir las características de los principales biomarcadores clínicos de síntomas no motores en la EP. Método. Se trata de una revisión bibliográfica utilizando las bases de datos PubMed / MEDLINE, SciELO, Science Direct. Los artículos fueron seleccionados según los criterios de inclusión: ensayos clínicos controlados aleatorizados, estudios cuasiexperimentales y revisiones sistemáticas que registraron el uso de biomarcadores de síntomas no motores en la EP. Resultados. La estrategia de búsqueda utilizada resultó en 705 títulos, donde se incluyeron un total de 21 estudios para su análisis. Más del $90 \%$ de los pacientes experimentan síntomas no motores durante el curso de la EP. Los principales síntomas no motores reportados involucran cambios en el sistema sensorial, cognitivo y cambios de personalidad. Conclusión. Los biomarcadores identificados en este estudio destacan el papel de la identificación de síntomas clínicos no motores en el diagnóstico temprano de la enfermedad de Parkinson y la comprensión de la etiología para guiar un tratamiento eficaz.
\end{abstract}

Palabras clave. Biomarcador; Enfermedad de Parkinson; Evaluación de síntomas

Trabalho realizado no Centro Universitário UNIFACISA, Campina Grande-PB, Brasil.

\title{
INTRODUÇÃO
}

A doença de Parkinson (DP) pode ser classificada como um transtorno multissistêmico com características motoras e não motoras. As características clínicas dessa doença refletem as mudanças neuroquímicas e neuroanatômicas com envolvimento não apenas do sistema dopaminérgiconigrostriatal, onde temos a deficiência de dopamina no corpo estriado levando ao desenvolvimento subsequente de sintomas motores, incluindo bradicinesia, tremor, rigidez e dificuldade na marcha, mas também de áreas do tronco cerebral serotoninérgico e noradrenérgico, regiões colinérgicas frontais e do tronco cerebral ${ }^{1,2}$. 
A DP uma das doenças neurodegenerativas mais prevalentes, é a segunda patologia neurodegenerativa mais comum do sistema nervoso central no mundo e o número de pacientes vem crescendo a cada ano. A sua prevalência chega a ser estimada entre 1 e 2 a cada 1.000 indivíduos. Sua prevalência pode sofrer variações de acordo com a idade, sexo e localização geográfica analisada, apesar de ser uma doença rara antes dos 50 anos, a DP chega a afetar cerca de $1 \%$ da população entre 65 a 74 anos e pode chegar a cerca de 4\% em indivíduos entre 85 a 94 anos $^{3}$.

O diagnóstico da DP está ancorado em critérios clínicos, que requerem a presença de bradicinesia e, pelo menos, um sintoma motor adicional de tremor, rigidez ou instabilidade postural. Alguns sintomas clínicos não motores como a hiposmia, distúrbios do sono e constipação intestinal podem marcar o início do curso da DP, identificados ainda na fase prodrômica, cerca de 5 a 10 anos antes do início das manifestações motoras ${ }^{4}$.

Estima-se que a taxa de erros de diagnóstico da DP pode variar de $10 \%$ a $50 \%$, essa falha pode estar relacionada ao fato de que comumente clínicos e pacientes não se atentam a buscar e relatar o aparecimento de sintomas não motores para detectar e diferenciar a DP de outros distúrbios do movimento com sintomas clínicos sobrepostos, para que assim a intervenção terapêutica tenha ação sobre os eventos patogênicos de forma precoce ${ }^{3,4}$.

Esses sintomas não motores podem se manifestar em qualquer estágio da doença, atualmente cerca de 30 
sintomas não motores têm sido estudados como biomarcadores na DP na tentativa de identificação da doença em estágios iniciais e consequente definição de um manejo capaz de impactar positivamente a qualidade de vida do indivíduo ${ }^{5}$. O objetivo desse trabalho foi identificar e discutir as características dos principais biomarcadores clínicos de sintomas não motores na doença de Parkinson.

\section{MÉTODO}

Realizou-se uma busca sistemática por artigos publicados em revistas indexadas nas bases de dados PubMed/MEDLINE, SciELO, LILACS e Science Direct por artigos publicados entre 2008 e 2018. Uma atualização na busca foi feita para identificar novas publicações entre 2018 e 2021. As palavras-chave utilizadas para a busca, "Biomarkers", "Nonmotor" e "Parkinson's Disease", seguiram a descrição dos termos MeSH/DeCS. A apresentação dos registros segue o PRISMA-Checklist (Preferred Reporting Items for Systematic Reviews and Meta-Analyses) ${ }^{6}$.

A presente pesquisa incluiu estudos que registraram 0 uso de biomarcadores de sintomas não motores para validar o diagnóstico precoce da Doença de Parkinson.

Os títulos e resumos, foram identificados e avaliados independentemente por 2 revisores na tela do computador, para selecionar aqueles que atendessem aos critérios de elegibilidade. Os estudos elegidos como relevantes e que geraram dúvidas foram retidos para uma análise posterior 
do texto na íntegra. Em casos de discordância no processo de seleção e na análise dos artigos, um terceiro avaliador participou da avaliação.

As buscas foram estendidas as referências dos artigos encontrados.

\section{RESULTADOS}

A estratégia de busca utilizada resultou em 428 títulos. Destes, 82 foram excluídos por duplicidade ou outras razões. Dos 346 estudos restantes, 193 não apresentaram os critérios de inclusão. Foram selecionados 153 estudos, avaliados através da análise do título e resumo, sendo 67 excluídos. Os 86 artigos retidos tiveram seus textos lidos na íntegra, dos quais 59 não apresentaram os critérios de elegibilidade, um total de 27 estudos foi incluído para síntese conforme descrito na Figura 1.

A amostra final reuniu $66 \%$ dos artigos publicados entre 2015-2021. A predominância da língua utilizada para comunicação foi a inglesa. 
Figura 1. Busca e seleção dos estudos para a revisão de acordo com o Preferred Reporting Items for Systematic Reviews and Meta-Analyses (PRISMA).

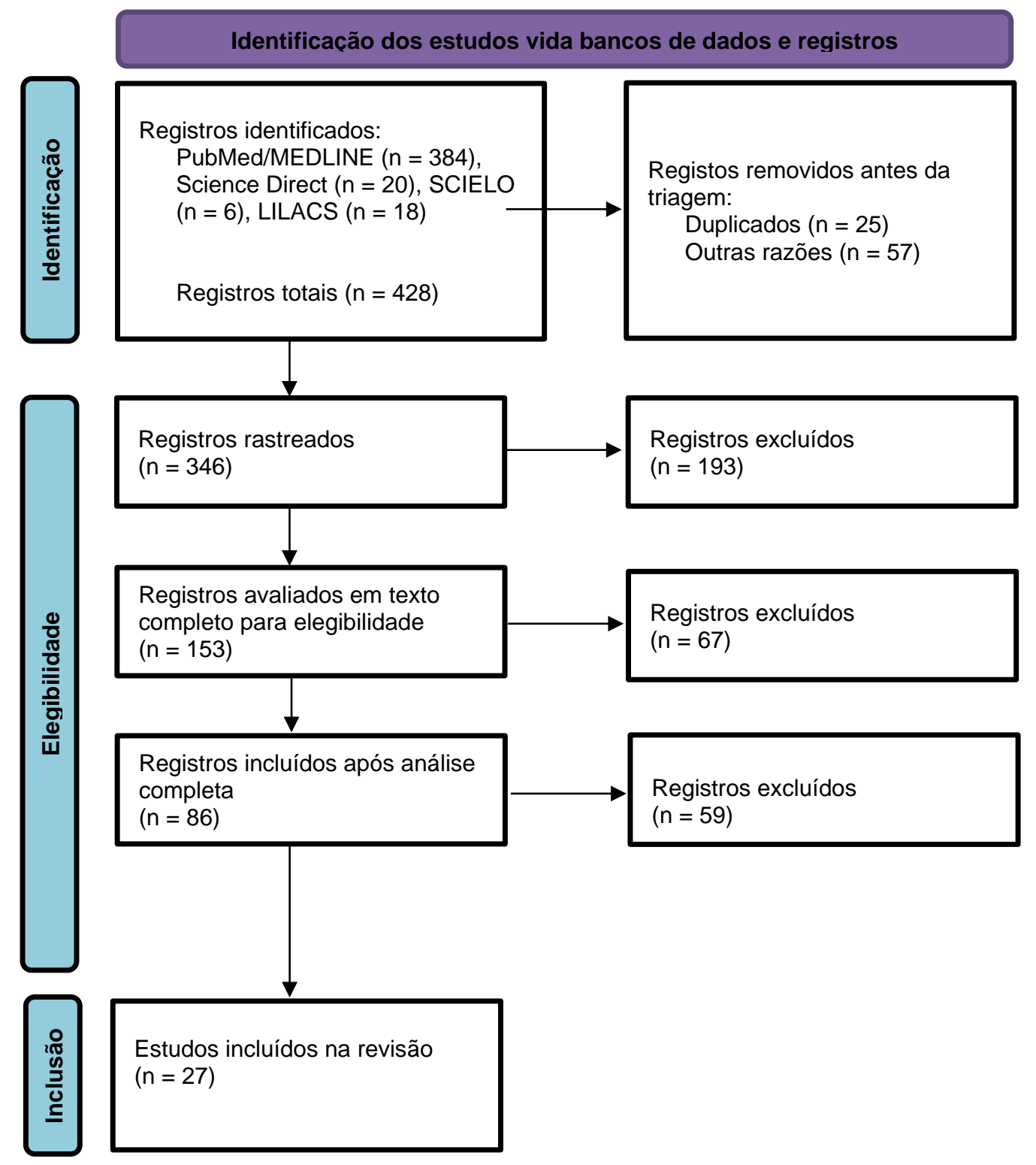

\section{DISCUSSÃO}

Um biomarcador pode ser definido como uma medida objetiva de indicador de um processo biológico normal, patológico ou proveniente de uma resposta farmacológica diante de uma interação, que pode ser usada como ferramenta para auxiliar a comunidade médica na tomada de 
decisão confirmando um diagnóstico e rastreando a progressão da doença7,8.

A dificuldade em firmar critérios de diagnóstico na DP é uma das barreiras para a validação de biomarcadores, tendo em vista que o início da doença pode apresentar sinais e sintomas tardios, já que diagnóstico clínico da DP geralmente ocorre apenas após a degeneração de um número substancial de neurônios ${ }^{8,9}$.

Alguns estudos têm verificado a validade de alguns biomarcadores não motores na DP, que podem ser baseados em fatores clínicos, de imagem, genéticos, proteômicos ou bioquímicos ou em uma combinação desses fatores, uma vez que a avaliação clínica para identificar indivíduos com DP pode ser mascarada por erros de diagnóstico no início da doença. Além disso, a progressão e a resposta ao tratamento são determinadas, sobretudo por meio de escalas clínicas subjetivas que se concentram quase unicamente nos sintomas motores ${ }^{10}$.

Existem uma grande diversidade de biomarcadores na DP e eles podem ser categorizados em quatro subgrupos principais: clínico, bioquímico, genético e de imagem. Quando um grupo é considerado isolado, a utilidade do biomarcador é comumente limitada, mas quando combinados e considerados coletivamente, os biomarcadores para a DP podem ser mais úteis ${ }^{7}$.

Mais de $90 \%$ dos pacientes experimentam sintomas não motores durante o curso da Doença de Parkinson, uma vez que apresentam degeneração em áreas extra-nigrais antes 
da perda dos neurônios nigrais. Os principais sintomas não motores compreendem a hiposmia, diminuição da acuidade visual, depressão, ansiedade, apatia, transtorno do sono e hipotensão ortostática, constipação, além de mudanças sutis na cognição e na personalidade. Embora essas características não sejam totalmente universais, elas estão presentes na maioria dos pacientes com DP antes que o diagnóstico seja confirmado ${ }^{1,4,10}$.

Apesar de serem sintomas comumente vistos na população geral, no entanto a presença de mais de uma dessas alterações são mais prevalentes em indivíduos com DP. A hiposmia foi o sintoma não motor mais visto quando foi feito o diagnóstico precoce da doença. Esta alteração chega a afetar pouco mais de $80 \%$ dos pacientes já diagnosticados com $\mathrm{DP}^{4,5,11}$. O período pré-clínico da DP pode se manifestar por pelo menos 20 anos antes das manifestações motoras, no entanto, apenas 0 comprometimento do sistema olfatório e a depressão podem preceder o aparecimento de manifestações motoras com um tempo preditivo menor ${ }^{12}$.

Um estudo realizado na Alemanha ${ }^{13}$, seguiu por três anos cerca de 1.800 homens e encontrou uma razão de risco relativo de 6,5 em participantes com hiposmia e 0 aparecimento da DP. As alterações olfatórias geralmente são bilaterais e parecem estar a associadas a erros no processamento central do olfato devido a diminuição do volume e da profundidade do sulco do bulbo olfatório ${ }^{14}$. 
Esses achados quando associados com alterações de biomarcadores de imagem e químicos, como por exemplo a presença do sinal reativo da a-sinucleína em regiões associadas ao olfato em exames de imagem, podem ser usados como uma boa ferramenta para o diagnóstico precoce da $\mathrm{DP}^{14,15}$, já que exames que a mucosa epitelial não revela nenhuma alteração quando a biopsia é o método de escolha para avaliar as queixas de déficit olfativo ${ }^{16}$.

A hiposmia quando a associada a hiperecogenicidade da substância nigra, pode apresentar uma boa sensibilidade e especificidade para DP, embora seu valor diagnóstico isolado possa ser limitado, o uso combinado da ultrassonografia da substância nigra e o teste olfativo específico é mais efetivo do que cada marcador isolado para distinguir pacientes com $\mathrm{DP}^{17,18}$.

Além de indicar o aparecimento da doença, esse marcador pode ser relacionado com a progressão e a severidade da doença, uma vez que pacientes com diagnóstico de DP estabelecido e que apresentam o declínio da função olfatória estão associados a quadros motores mais graves $^{19}$.

A desordem do comportamento do sono REM de origem idiopática possui uma taxa de conversão para doenças neurodegenerativas, demência primária e DP de até 33\%. A combinação de outros sintomas não motores visto junto a alteração do sono REM de origem idiopática pode chegar a apresentar uma taxa de conversão maior que $80 \%$ em 5 $\operatorname{anos}^{20-22}$. 
Esse tipo de distúrbio pode assumir diversas formas clínicas já que os neurotransmissores que regulam a atividade do sono e outras funções associadas (dopamina, noradrenalina e 5-HT), estão afetados na DP. A detecção precoce e manejo adequado dos distúrbios do sono são fundamentais para evitar o impacto negativo da doença na qualidade de vida 5,14 .

A depressão é a morbidade neuropsiquiátrica mais comum na DP podendo chegar a afetar cerca de 40-50\% dos pacientes, impactando diretamente de forma negativa a qualidade de vida. O mecanismo precursor da depressão na DP é complexo e pode envolver fatores biológicos, psicossociais e terapêuticos. Dentro do mecanismo biológico essas alterações podem envolver os sistemas dopaminérgico e, principalmente, o serotoninérgico com uma diminuição do receptor 5-HT na fenda sináptica, os defeitos nesses sistemas estão ligados a depressão assim como a DP23.

No entanto, por se tratar de um mecanismo complexo que pode causado pelo mecanismo que desencadeia a DP ou por reação aos sintomas associados, não há evidência estabelecida para a conversão da depressão em algum tipo de neurodegeneração ${ }^{24}$.

A constipação intestinal é um sintoma que chega a afetar cerca de $28 \%$ a $61 \%$ dos pacientes com DP e pode representar um risco relativo de 2,7 para homens que apresentem motilidade intestinal lenta, com uma frequência de até uma vez ao dia, quando comparado com homens de função intestinal mais frequente ${ }^{25}$. 
A disfunção intestinal pode acometer todo o tubo digestivo e ainda pode ser percebida na presença de salivação excessiva, disfagia e esvaziamento gástrico lentificado, ocasionando crescimento bacteriano e complicações decorrentes desse fato. Essas alterações podem acontecer quando existe a alteração neuromoduladora no núcleo dorsal do nervo vago, responsável por organizar a função intestinal ${ }^{14}$.

A presença de grandes quantidades de a-sinucleína nas biópsias de glândulas salivares, reto e cólon na presença de sintomas não motores já mencionados, têm sido associadas ao diagnóstico da DP ainda na fase pré-clínica. No entanto a presença dessa substância precisa ser avaliada com cuidado, pois em indivíduos sadios os níveis de a-sinucleína aumentam com a idade mesmo na ausência de neurodegeneração $0^{5,14,26}$.

Um estudo analisou a massa cefálica de 245 indivíduos post-mortem e relatou uma associação positiva entre o ritmo intestinal e o aparecimento dos corpos de Lewy, onde $24,1 \%$ dos indivíduos apresentaram durante a vida menos de um evento de evacuação por dia, já os que apresentavam uma frequência superior a um evento por dia representavam apenas $6,5 \% 25,27$.

Apesar dos sintomas motores melhorarem a especificidade, a sensibilidade do diagnóstico da DP, quando utilizados de forma isolada, ainda não é claramente estabelecida, tornando ainda sua usabilidade dependente de associações com imagens e padrões bioquímicos. Assim, 
esses critérios adicionais não substituem os critérios clínicos existentes, ao invés disso, eles os suplementariam para melhor identificação dos indivíduos susceptíveis para o fechamento do diagnóstico precoce?.

\section{CONCLUSÃO}

Os sintomas não motores da DP podem, quando bem avaliados, se tornar uma excelente oportunidade para identificar populações com maior risco de DP além de entender a etiologia precoce dessa doença e definir estratégias de tratamento que impactem positivamente na qualidade de vida do doente. No entanto, nenhum dos sintomas não-motores discutidos nessa revisão tem especificidade e sensibilidade suficientes para, de forma isolada, qualificar o rastreamento do risco de DP na população.

Alguns biomarcadores clínicos de sintomas não motores ainda são postos a prova diante de estudos clínicos e questionados devido à presença desses sinais e sintomas sendo compartilhados com outras doenças e síndromes, apesar de desafiador esses estudos podem impactar positivamente no diagnóstico e tratamento da DP.

\section{REFERÊNCIAS}

1.Goldman JG, Postuma R. Premotor and nonmotor features of Parkinson's disease. Curr Opin Neurol 2014;27:434-41. https://doi.org/10.1097/WC0.0000000000000112

2.Sharma VD, Lyons KE, Pahwa R. Amantadine extended-release 
capsules for levodopa-induced dyskinesia in patients with Parkinson's disease. Ther Clin Risk Manag 2018;14:665-73. https://doi.org/10.2147/TCRM.S144481

3. Wang J, Hoekstra JG, Zuo C, Cook TJ, Zhang J. Biomarkers of Parkinson's disease: Current status and future perspectives. Drug Discov Today

2013; 18:155-62.

https://doi.org/10.1016/j.drudis.2012.09.001

4. Mahlknecht P, Seppi K, Poewe W. The concept of prodromal Parkinson's disease. J Parkinsons Dis 2015;5:681-97. https://doi.org/10.3233/JPD-150685

5.Kumaresan M, Khan S. Spectrum of Non-Motor Symptoms in Parkinson's Disease. Cureus 2021;13:e13275. https://doi.org/10.7759/cureus.13275

6. Shamseer L, Moher D, Clarke M, Ghersi D, Liberati A, Petticrew M, et al. Preferred reporting items for systematic review and meta-analysis protocols (prisma-p) 2015: Elaboration and explanation. BMJ 2015;349:g7647. http://doi.org/10.1136/bmj.g7647

7.Delenclos M, Jones DR, McLean PJ, Uitti RJ. Biomarkers in Parkinson's disease: Advances and strategies. Park Relat Disord 2016;22:S10610. http://doi.org/10.1016/j.parkreldis. 2015.09.048

8. Miller DB, O'Callaghan JP. Biomarkers of Parkinson's disease: Present and future. Metabolism 2015;64:S40-6. http://doi.org/10.1016/j.metabol.2014.10.030.

9. Costa MFBNA, Reisdorfer E, Kempfer SS, Fernandes GCM, Porporatti $A L$, Canto GDL. Diagnostic validity of biomarkers in Parkinson's Disease: systematic review and meta-analysis. Rev Bras Enferm 2018;71:3074-83. https://doi.org/10.1590/0034-7167-2017-0822

10.Siderowf A, Lang AE. Premotor Parkinson's disease: Concepts and definitions. Mov Disord 2012;27:608-

16. http://doi.org/10.1002/mds.24954

11.Swallow DMA, Lawton MA, Grosset KA, Malek N, Smith CR, Bajaj $\mathrm{NP}$, et al. Variation in recent onset Parkinson's disease: Implications for prodromal detection. J Parkinsons Dis 2016;6:289-300. http://doi.org/10.3233/JPD-150741

12.Savica R, Rocca WA, Ahlskog JE. When does Parkinson disease start? Arch Neurol 2010;67:798-801. http://doi.org/10.1001/archneurol.2010.135

13.Berg D, Godau J, Seppi K, Behnke S, Liepelt-Scarfone I, Lerche S, et al. The PRIPS study: Screening battery for subjects at risk for Parkinson's disease. Eur J Neurol 2013;20:102-8. http://doi.org/10.1111/j.1468-1331.2012.03798.x

14.Schapira AHV, Chaudhuri KR, Jenner P. Non-motor features of Parkinson disease. Nat Rev Neurosci 2017;18:435-50. https://doi.org/10.1038/nrn.2017.62

15.Lotankar S, Prabhavalkar KS, Bhatt LK. Biomarkers for Parkinson's Disease: Recent Advancement. Neurosci Bull 2017;33:585-97. http://doi.org/10.1007/s12264-017-0183-5

16.Chase BA, Markopoulou K. Olfactory Dysfunction in Familial and Sporadic Parkinson's Disease. Front Neurol 2020;11:1-11. 
https://doi.org/10.3389/fneur.2020.00447

17.Tunc S, Graf J, Tadic V, Brüggemann N, Schmidt A, Al-Khaled M, et al. A population-based study on combined markers for early Parkinson's disease. Mov Disord 2015;30:531-7. http://doi.org/10.1002/mds.26100

18. López Hernández N, García Escrivá A, Shalabi Benavent M. Valor de la evaluación combinada de olfación e hiperecogenicidad de sustancia negra en el diagnóstico de la enfermedad de Parkinson. Neurología 2015;30:496-501. https://doi.org/10.1016/j.nrl.2014.03.010

19. He R, Zhao Y, He Y, Zhou Y, Yang J, Zhou X, et al. Olfactory Dysfunction Predicts Disease Progression in Parkinson's Disease: A Longitudinal Study. Front Neurosci 2020;14:1-9. https://doi.org/10.3389/fnins.2020.569777

20.Postuma RB, Iranzo A, Hogl B, Arnulf I, Ferini-Strambi L, Manni R, et al. Risk factors for neurodegeneration in idiopathic rapid eye movement sleep behavior disorder: A multicenter study. Ann Neurol 2015;77:830-9. http://doi.org/10.1002/ana.24385

21.Postuma RB, Gagnon JF, Bertrand JA, Génier Marchand D, Montplaisir JY. Parkinson risk in idiopathic REM sleep behavior disorder: Preparing for neuroprotective trials. Neurology 2015;84:1104-13. http://doi.org/10.1212/WNL.0000000000001364

22.Postuma RB. Prodromal Parkinson's disease - Using REM sleep behavior disorder as a window. Park Relat Disord 2014;20(suppl 1):S1-4. https://doi.org/10.1016/S1353-8020(13)00400-8

23.Sharma S, Moon CS, Khogali A, Haidous A, Chabenne A, Ojo C, et al. Biomarkers in Parkinson's disease (recent update). Neurochem Int 2013;63:201-29. https://doi.org/10.1016/j. neuint.2013.06.005

24.Skorvanek M, Feketeova E, Kurtis MM, Rusz J, Sonka K. Accuracy of rating scales and clinical measures for screening of rapid eye movement sleep behavior disorder and for predicting conversion to Parkinson's disease and other synucleinopathies. Front Neurol 2018;9:1-18. https://doi.org/10.3389/fneur.2018.00376

25. Abbott RD, Petrovitch H, White LR, Masaki KH, Tanner CM, Curb JD, et al. Frequency of bowel movements and the future risk of Parkinson's disease. Neurology 2001;57:456-62. http://doi.org/10.1212/wnl.57.3.456

26. Fayyad M, Salim S, Majbour N, Erskine D, Stoops E, Mollenhauer B, et al. Parkinson's disease biomarkers based on a-synuclein. J Neurochem 2019;150:626-36. https://doi.org/10.1111/jnc.14809

27. Pouclet $H$, Lebouvier T, Coron E, Bruley des Varannes S, Rouaud T, Roy $M$, et al. A comparison between rectal and colonic biopsies to detect Lewy pathology in Parkinson's disease. Neurobiol Dis 2012;45:305-9. https://doi.org/10.1016/j.nbd.2011.08.014 